\title{
Risk Factors of Synchronous Inguinal Lymph Nodes Metastasis for Lower Rectal Cancer Involving the Anal Canal
}

\author{
Renjie Wang ${ }^{1,29}$, Peng Wu ${ }^{1,29}$, Debing Shi ${ }^{1,2}$, Hongtu Zheng ${ }^{1,2}$, Liyong Huang ${ }^{1,2}$, Weilie Gu ${ }^{1,2}$, Ye Xu ${ }^{1,2}$, \\ Sanjun Cai ${ }^{1,2}$, Guoxiang Cai ${ }^{1,2 *}$
}

1 Department of Colorectal Surgery, Fudan University Shanghai Cancer Center, Shanghai, China, 2 Department of Oncology, Shanghai Medical College, Fudan University, Shanghai, China

\begin{abstract}
Purpose: The aim of the study is to identify the risk factors of synchronous ILN metastasis for lower rectal cancer involving the anal canal.

Methods: Patients with lower rectal cancer who underwent radical resection at the Fudan University Shanghai Cancer Center were retrospectively analyzed. The synchronous ILN metastasis was defined as the metastasis occurring within 6 months after the diagnosis of rectal cancer. Patients' gender, age, tumor diameter, dentate line invasion, differentiation level, histological type, depth of invasion, perirectal LN metastasis, lymphovascular invasion or perineural invasion were analyzed in the study. The correlation between synchronous ILN involvement and clinicopathological features were analyzed with Chi-square test/fisher's exact test. Variables with $\mathrm{p}<0.05$ in univariate analysis were then analyzed in a multivariate logistic model. Odds ratio (OR) along with $95 \%$ confidence intervals $(95 \% \mathrm{Cl})$ were calculated.
\end{abstract}

Results: A total of 325 patients (182 men and 143 women) with lower rectal cancer met the criteria and were enrolled in the study. Among them, 20 patients (6.2\%) had synchronous ILN metastasis. Both univariate and multivariate analysis showed the invasion of the dentate line had a strong correlation with synchronous ILN metastasis with the odds ratio (OR) of 23.558 [95\% confidence interval $(\mathrm{Cl}) 6.380-86.982](p<0.001)$. The presence of lymphovascular invasion also showed a significant correlation synchronous ILN metastasis with odds ratio (OR) of 5.260 [95\% confidence interval $(C I) 1.818-15.212](p=0.002)$.

Conclusions: The invasion of dentate line and lymphovascular invasion are two independent risk factors of inguinal lymph node metastasis for lower rectal cancer involving the anal canal.

Citation: Wang R, Wu P, Shi D, Zheng H, Huang L, et al. (2014) Risk Factors of Synchronous Inguinal Lymph Nodes Metastasis for Lower Rectal Cancer Involving the Anal Canal. PLoS ONE 9(11): e111770. doi:10.1371/journal.pone.0111770

Editor: John Souglakos, University General Hospital of Heraklion and Laboratory of Tumor Cell Biology, School of Medicine, University of Crete, Greece

Received April 5, 2014; Accepted October 1, 2014; Published November 19, 2014

Copyright: (C) 2014 Wang et al. This is an open-access article distributed under the terms of the Creative Commons Attribution License, which permits unrestricted use, distribution, and reproduction in any medium, provided the original author and source are credited.

Data Availability: The authors confirm that all data underlying the findings are fully available without restriction. All relevant data are within the paper.

Funding: This study was supported by grants from the National Natural Science Foundation of China (No. 81001055), Shanghai Pujiang Program (No. 13PJD008) National High Technology Research and Development Program (863 Program, No. 2012AA02A506) and Shanghai Shenkang Program (No. SHDC12014206 and No. SHDC12012120). The funders had no role in study design, data collection and analysis, decision to publish, or preparation of the manuscript.

Competing Interests: The authors have declared that no competing interests exist.

* Email: gxcai@fudan.edu.cn

9 These authors contributed equally to this work.

\section{Introduction}

Inguinal lymph nodes (ILN) metastases from rectal cancer are relatively rare [1,2]. They are believed to arise from advanced primary lesions with proximal lymphatic obstruction which results retrograde nodal spread, or from recurrent disease in the pelvis or perineum [3]. In a retrospective study of 32 patients with ILN metastases from rectal cancer, $0 \% 5$-year overall survival was observed, which showed poor prognosis of ILN metastases in rectal cancer. Lymphatic spread from tumors located in the lower rectum are also complex and unpredictable [2]. Therefore, the prevention for ILN metastases is extremely critical for patients with lower rectal cancer. Compared to penile cancer and perineal cancer, fewer studies were related with ILN metastasis in lower rectal cancer. In this study, we performed a retrospective study of a cohort of lower rectal cancer patients and analyzed the potential predictors of synchronous ILN metastasis.

\section{Materials and Methods}

Patients with lower rectal cancer who underwent abdominal perineal resection (APR) at the Fudan University Shanghai Cancer Center from September 1986 to July 2013 were reviewed in this study. None of the patients received any preoperativechemoradiotherapy at operation. Written informed consent was obtained from all study participants adhering to the local ethical guidelines prior to specimen collection. The study protocol and consent 
procedure were approved the Ethics Committee of the Shanghai Cancer Center, Fudan University.

All the patients met the criteria as follows: (1) the lower edge of the tumor located less than $2 \mathrm{~cm}$ proximal to the dentate line on digital rectal examination and then was confirmed by postoperative pathological record; (2) having complete medical records including demographic information, clinical and pathological data, operation notes, and follow-up results; and (3) having at least 6 months follow-up time after the operation.

Patients who (1) had ILN metastasis occurring 6 months after the diagnosis of rectal cancer (61 patients); (2) had incomplete medical records (18 patients); (3) had other histological type including squamous carcinoma, carcinoid and melanoma (5 patients) were all excluded from the study. ILN Metastases were suspected either by palpable lymph nodes or by image results before or after the surgical procedure. The synchronous ILN metastasis was defined as the metastasis occurring within 6 months after the diagnosis of anorectal cancer and confirmed by pathology with the inguinal lymph nodes resection or biopsy or fine needle aspiration. The cancer staging was based on the American Joint Committee on Cancer 7th edition.

Patient's gender, age, tumor diameter, dentate line invasion, differentiation level, histological type, depth of invasion, perirectal LN metastasis, lymphovascular invasion or perineural invasion were analyzed in the study. ILN metastasis was not defined as perirectal LN metastasis.

The correlation between synchronous ILN involvement and clinicopathological features were analyzed with Chi-square test/ fisher's exact test. Variables with $\mathrm{p}<0.05$ in univariate analysis were then analyzed in a multivariate logistic model (using binary logistic, step backward method). Values of $\mathrm{p}<0.05$ was considered statistically significant, and odds ratio (OR) along with $95 \%$ confidence intervals (95\% CI) were calculated. All statistical analysis were performed by SPSS, version 19.0.0 (SPSS, Inc., Chicago, IL, USA).

\section{Results}

\section{General information of patients}

A total of 325 patients (182 men and 143 women) with lower rectal cancer met the criteria and were enrolled in the study. The median age at the time the diagnosis of rectal cancer was 56 years (range, 25-86 years). The median maximum diameter of the tumor was $36.0 \mathrm{~mm}$ (range, 8-100 mm). Among them, 20 patients $(6.2 \%)$ were confirmed with synchronous ILN metastasis (Table 1). The clinical and pathological data and postoperative treatment information of the 20 patients with ILN metastasis is shown in Table 2.

\section{Univariate analysis of risk factors for lower rectal cancer involving the anal canal}

The associations between synchronous ILN metastasis and clinicopathological features are shown in Table 3. The univariate analysis showed factors including dentate line invasion, perirectal LN metastasis and lymphovascular invasion were significantly related to the synchronous ILN metastasis. No significant correlation was seen between synchronous ILN metastasis and patients' gender, age, tumor diameter, histological type, $\mathrm{T}$ stage or presence of perineural invasion.

\section{Multivariate analysis of risk factors for lower rectal cancer involving the anal canal}

Variables with $\mathrm{p}<0.05$ in univariate analysis were then analyzed. Multivariate logistic analysis was performed (Table 4) with the factor including dentate line invasion, perirectal LN metastasis and lymphovascular invasion. The invasion of the dentate line showed a strong correlation with synchronous ILN metastasis with the odds ratio (OR) of 23.558 [95\% confidence interval (CI) 6.380-86.982] $(\mathrm{p}<0.001)$. The presence of lymphovascular invasion also showed a significant correlation synchronous ILN metastasis with odds ratio (OR) of $5.260 \quad[95 \%$ confidence interval (CI) 1.818-15.212] $(\mathrm{p}=0.002)$. The results indicated invasion of the dentate line and lymphovascular invasion are two independent risk factors for lower rectal cancer involving the anal canal.

\section{Discussion}

The rectum is divided into upper and lower segments according to the relative location from the peritoneal reflection by convention. In Japan, the lower rectum is further divided into two subregions, i.e. 'Rb tumors' and 'Rp tumors.' An 'Rb tumor' indicates a tumor located in the lower rectum without any invasion of the dentate line. An 'Rp tumor' indicates a tumor invades the dentate line [4]. In a Japanese study of 156 lower rectal cancer patients, $28 \%$ (7/25) of 'Rp tumor' patients had ILN metastasis. 'Rp tumor' was found associated with high rate of ILN metastasis, and also had worse prognosis and local recurrence than ' $R b$ tumor'. And the presence of poorly differentiated or mucinous adenocarcinoma is a risk factor of local recurrence for $25 \mathrm{Rp}$ tumor' patients [5]. In our studies, there are 83 patientsmet the criteria of ' $\mathrm{Rp}$ tumor.' And our results showed 'Rp tumor', invading the dentate line, is an independent risk factor for lower rectal cancer involving the anal canal. This can be explained that in a tumour that originates above the dentate line the lymphatics drain mostly to the mesenteric lymph nodes (MLN) and lateral lymph nodes (LLN), while below the dentate line the lymphatics drain mostly to the inguinal lymph nodes (ILN) [6]. Moreover, in our study, the presence of lymphovascular invasion of lower rectal cancer is shown to be another independent risk factor. The result is consistent with the lymphatic anatomy. Also, we found no significant correlation between synchronous ILN metastasis and patients' gender, age, tumor size, histological type, $\mathrm{T}$ stage or presence of perineural invasion, which was never revealed in other studies.

For all patients underwent digital rectal examination, their tumor location will be again confirmed by postoperative pathological record. As for the tumors which overlap the anorectal junction, the determination of the anatomy can be ambiguous. In our study, we used the classification from AJCG. According to AJCC, if epicenter of a tumor is located more than $2 \mathrm{~cm}$ proximal to the dentate line or proximal to the anorectal ring on digital rectal examination, such tumor should be classified as rectal cancer [7].

Compared to rectal cancer, the ILN metastasis is more common in anal canal carcinoma with 5-25\% patients [8-12]. In a cohort study of 206 lower rectal cancer patients, Bebenek Marek et al reported ILN metastasis with only $2.9 \%$ (6/206) patients [13].

Although there are few reports about ILN metastasis for rectal cancer, the outcome of poor prognosis of ILN metastasis for rectal cancer is clear [13,14]. Therefore, prevention or early diagnosis of ILN metastasis for lower rectal cancer is very critical. Although PET-CT scan is useful for staging and finding metastatic lesions, according to NCGN guidelines version 3.2014 for rectal cancer, it does not supplant a contrast-enhanced diagnositic CT scan. PETCT should only be used to evaluate an equivocal finding on a contrast-enhanced CT scan or in patients with strong contraindications to IV contrast. And for the equivocal findings on a 
Table 1. General information of patients $(n=325)$.

\begin{tabular}{|c|c|}
\hline Characteristic & Cases $(\%)(n=325)$ \\
\hline \multicolumn{2}{|l|}{ Gender } \\
\hline Male & $182(56.0 \%)$ \\
\hline Female & $143(44.0 \%)$ \\
\hline Median age (years) at diagnosis (range) & $56.0(25-86)$ \\
\hline \multicolumn{2}{|l|}{ Dentate line invasion } \\
\hline Yes & $89(27.4 \%)$ \\
\hline No & $236(72.6 \%)$ \\
\hline Maximum diameter $(\mathrm{mm})$ of tumor (range) & $36.0(8-100)$ \\
\hline \multicolumn{2}{|l|}{ Histological type } \\
\hline Adenocarcinoma & $264(81.2 \%)$ \\
\hline Mucinous & $57(17.6 \%)$ \\
\hline Signet-cell & $4(1.2 \%)$ \\
\hline \multicolumn{2}{|l|}{ Differentiation } \\
\hline Poor & $107(32.9 \%)$ \\
\hline Moderate & $215(66.2 \%)$ \\
\hline Well & $3(0.9 \%)$ \\
\hline \multicolumn{2}{|l|}{ Depth of invasion } \\
\hline $\mathrm{T} 1$ & $9(2.8 \%)$ \\
\hline T2 & $122(37.5 \%)$ \\
\hline T3 & $56(17.2 \%)$ \\
\hline T4 & $138(42.5 \%)$ \\
\hline \multicolumn{2}{|l|}{ Perirectal LNM } \\
\hline Yes & $146(44.9 \%)$ \\
\hline No & $179(55.1 \%)$ \\
\hline \multicolumn{2}{|l|}{ Lymphovascular invasion } \\
\hline Yes & $70(21.5 \%)$ \\
\hline No & 255 (78.5\%) \\
\hline \multicolumn{2}{|l|}{ Perineural invasion } \\
\hline Yes & $78(24.0 \%)$ \\
\hline No & 247 (76.0\%) \\
\hline \multicolumn{2}{|l|}{ Synchronous ILN metastasis } \\
\hline Yes & $20(6.2 \%)$ \\
\hline No & 305 (93.8\%) \\
\hline
\end{tabular}

contrast-enhanced CT scan, aspiration or biopsy is still the gold standard, the specificity and positive predictive value of PET-CT is only $83 \%$ and $43 \%$ respectively [15]. In our institution, ILN dissection and postoperative ILN irradiation is the conventional treatment for synchronous ILN metastasis for lower rectal cancer. In patients confirmed with synchronous ILN metastasis, 12 patients (60\%) received ILN dissection, 3 patients (15\%) received postoperative ILN irradiation, 1 patient $(5 \%)$ received both irradiation and ressection and 4 patients (20\%) were given palliative chemotherapy because of systemic metastases (Table 2). The role of preventive treatment for ILN metastasis for lower rectal cancer has been not well defined yet. The complication of ILN dissection includes lymphoedema and lymphocele [16-18] and prophylactic ILN dissection is also not a routine treatment for lower rectal cancer. In our study, the dentate line invasion and lymphovascular invasion were shown to be critical risk factors for synchronous ILN metastasis for lower rectal cancer. Therefore, for those patients with those risk factors, prophylactic ILN dissection could be considered to prevent the ILN metastasis. As for the radiotherapy, there is little information about the preventive irradiation either. A study from M.D. Anderson claimed that inguinal nodal failure in rectal cancer patients with anal canal involvement treated with neoadjuvant or adjuvant chemoradiation is not high enough to justify routine elective groin irradiation [19]. In 2010 clinical practice guideline for rectal cancer, experts from ESMO believed medial inguinal nodes need only be included prophylactically for radiotherapy when the tumor grows at or below the dentate line [20].

Moreover, we had to admit there was still limitation in our study. The sample group is small with only 20 patients included. Therefore, a larger size of sample with multiple centers is called for further study. And patients with metachronous ILN metastasis (ILN metastasis occurring over 6 months after the diagnosis of rectal cancer) will be analyzed in the following study, which would 


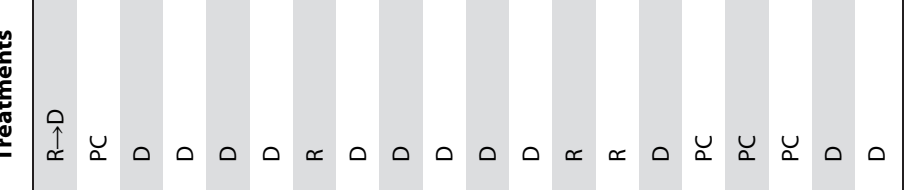

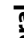

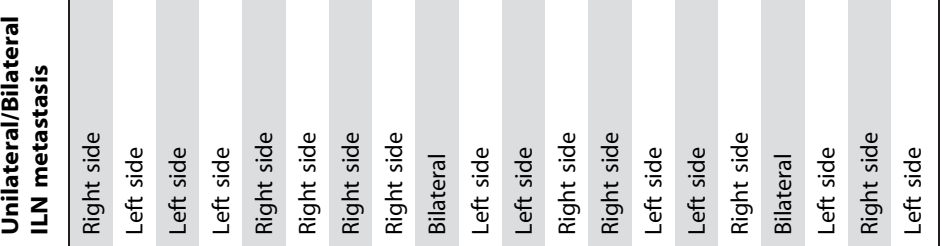

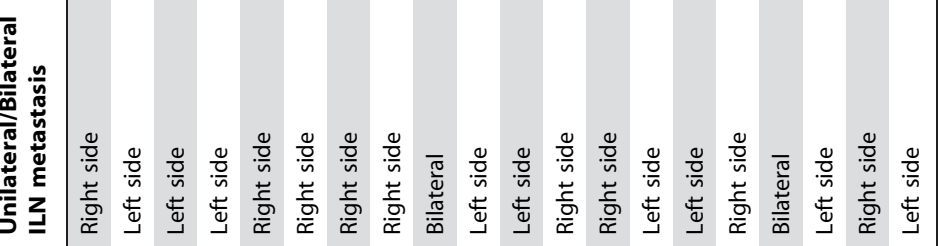

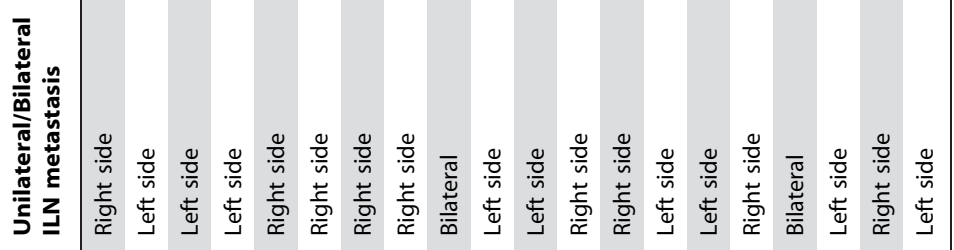

政

ב.

|

$\sum \frac{T}{ \pm}$

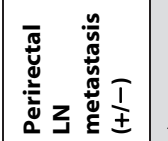

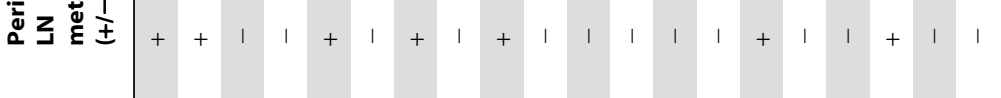

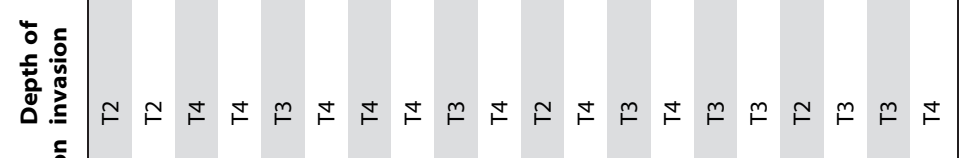

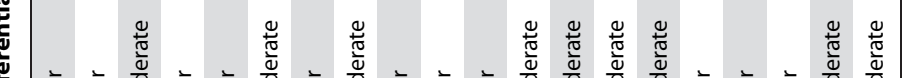

立

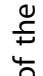

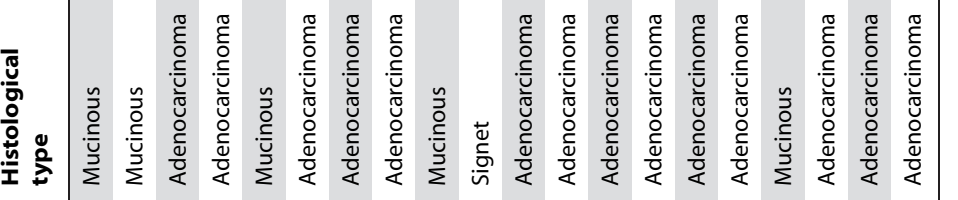

离

है

$\frac{\mathrm{g}}{\mathrm{og}} \overline{\mathrm{g}}$

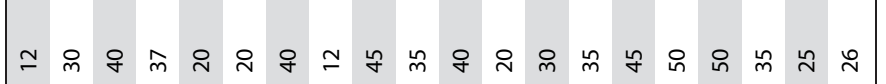

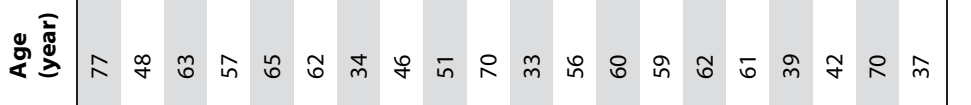

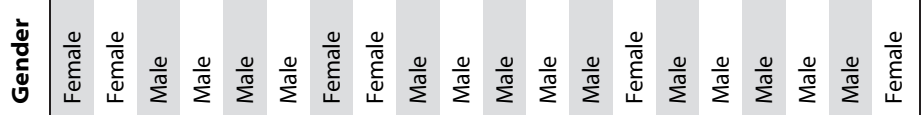

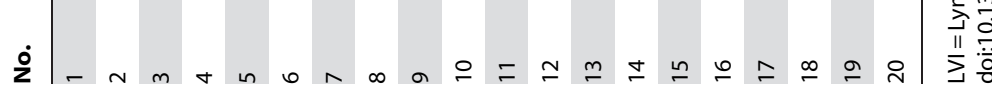


Table 3. Association between clinicopathological factors and synchronous ILN metastasis.

\begin{tabular}{|c|c|c|c|}
\hline \multirow[t]{2}{*}{ Characteristic } & \multicolumn{2}{|c|}{ Synchronous ILN Metastasis } & \multirow[t]{2}{*}{$P$ Value } \\
\hline & Yes $(n=20)$ & No $(n=305)$ & \\
\hline Gender & & & 0.247 \\
\hline Male & $14(70.0 \%)$ & 168 (55.1\%) & \\
\hline Female & $6(30.0 \%)$ & $137(44.9 \%)$ & \\
\hline Age & & & 0.635 \\
\hline$\geq 60$ years & $9(45.0 \%)$ & 114 (37.4\%) & \\
\hline$<60$ years & $11(55.0 \%)$ & $191(62.6 \%)$ & \\
\hline Dentate line invasion & & & $<0.001$ \\
\hline Yes & $17(85.0 \%)$ & $72(23.6 \%)$ & \\
\hline No & $3(15.0 \%)$ & 233 (76.4\%) & \\
\hline Maximum diameter & & & 0.816 \\
\hline$\geq 30 \mathrm{~mm}$ & $11(55.0 \%)$ & $180(59.0 \%)$ & \\
\hline$<30 \mathrm{~mm}$ & $9(45.0 \%)$ & 125 (41.0\%) & \\
\hline Histological type & & & 0.140 \\
\hline Adenocarcinoma & $14(70.0 \%)$ & $250(82.0 \%)$ & \\
\hline Mucinous & $5(25 \%)$ & $52(17 \%)$ & \\
\hline Signet & $1(5 \%)$ & $3(1 \%)$ & \\
\hline Differentiation & & & 0.102 \\
\hline Poor & $11(55.0 \%)$ & 96 (31.5\%) & \\
\hline Moderate & $9(45.0 \%)$ & $206(67.5 \%)$ & \\
\hline Well & $0(0 \%)$ & $3(1 \%)$ & \\
\hline T stage & & & 0.062 \\
\hline T1-2 & $4(20.0 \%)$ & $127(41.6 \%)$ & \\
\hline T3-4 & $16(80.0 \%)$ & 178 (58.4\%) & \\
\hline Perirectal LNM & & & 0.022 \\
\hline Positive & $14(70.0 \%)$ & $132(43.3 \%)$ & \\
\hline Negative & $6(30.0 \%)$ & $173(56.7 \%)$ & \\
\hline LVI & & & 0.019 \\
\hline Yes & $9(45.0 \%)$ & $61(20.0 \%)$ & \\
\hline No & $11(55.0 \%)$ & $244(80.0 \%)$ & \\
\hline \multicolumn{4}{|l|}{ Perineural invasion } \\
\hline Yes & $6(30.0 \%)$ & $72(23.6 \%)$ & 0.705 \\
\hline No & $14(70.0 \%)$ & 233 (76.4\%) & \\
\hline
\end{tabular}

doi:10.1371/journal.pone.0111770.t003

Table 4. Multivariate analysis.

\begin{tabular}{lllll}
\hline Characteristic & $\mathbf{n}$ & $\mathbf{O R}$ & $\mathbf{9 5 \%} \mathbf{C l}$ & $\mathbf{P}$ \\
\hline $\begin{array}{l}\text { Dentate line invasion } \\
\text { No (Referent) }\end{array}$ & 236 & 1.0 & & $<0.001$ \\
Yes & 89 & 22.759 & $6.137-84.404$ & \\
Lymphovascular invasion & & & & 0.015 \\
No (Referent) & 255 & 1.0 & $1.317-13.122$ & \\
Yes & 70 & 4.157 & & 0.034 \\
Perirectal LNM & & & & \\
No (Referent) & 179 & 1.0 & $0.562-5.467$ & \\
Yes & 146 & 1.753 & & \\
\hline doi:10.1371/journal.pone.0111770.t004 & & &
\end{tabular}


give alternative approach in prophylactic treatment of ILN metastasis for lower rectal cancer involving the anal canal.

\section{Conclusion}

The invasion of dentate line and lymphovascular invasion are two independent risk factors of inguinal lymph node metastasis for lower rectal cancer involving the anal canal. For patients with these risk factors, radiological assessment for inguinal region and biopsy for inguinal lymphadenopathy are recommended.

\section{Acknowledgments}

This study was supported by grants from the National Natural Science Foundation of China (No. 81001055), Shanghai Pujiang Program

\section{References}

1. Graham RA, Hohn DC (1990) Management of inguinal lymph node metastases from adenocarcinoma of the rectum. Diseases of the colon and rectum 33:212 216.

2. Luna-Perez P, Corral P, Labastida S, Rodriguez-Coria D, Delgado S (1999) Inguinal lymph node metastases from rectal adenocarcinoma. Journal of surgical oncology 70: 177-180.

3. Grinnell RS (1942) The Lymphatic And Venous Spread Of Carcinoma Of the Rectum. Annals of surgery 116: 200-216.

4. Mori T, Takahashi K, Yamaguchi T, Matsumoto H (2003) [General rules for management of cancer of the colon and the rectum]. Nihon rinsho Japanese journal of clinical medicine 61 Suppl 7: 255-261.

5. Hamano T, Homma Y, Otsuki Y, Shimizu S, Kobayashi H, et al. (2010) Inguinal lymph node metastases are recognized with high frequency in rectal adenocarcinoma invading the dentate line. The histological features at the invasive front may predict inguinal lymph node metastasis. Colorectal disease: the official journal of the Association of Coloproctology of Great Britain and Ireland 12: e200-205.

6. Frost DB, Richards PC, Montague ED, Giacco GG, Martin RG (1984) Epidermoid cancer of the anorectum. Cancer 53: 1285-1293.

7. Edge SB, Compton CC (2010) The American Joint Committee on Cancer: the 7th edition of the AJCC cancer staging manual and the future of TNM. Annals of surgical oncology 17: 1471-1474.

8. Boman BM, Moertel CG, O'Connell MJ, Scott M, Weiland LH, et al. (1984) Carcinoma of the anal canal. A clinical and pathologic study of 188 cases. Cancer 54: 114-125.

9. Fuchshuber PR, Rodriguez-Bigas M, Weber T, Petrelli NJ (1997) Anal canal and perianal epidermoid cancers. Journal of the American College of Surgeons 185: 494-505.

10. Papillon J, Montbarbon JF (1987) Epidermoid carcinoma of the anal canal. A series of 276 cases. Diseases of the colon and rectum 30: 324-333.
(No. 13PJD008), National High Technology Research and Development Program (863 Program, No. 2012AA02A506) and Shanghai Shenkang Program (No. SHDC12014206 and No. SHDC12012120). We would like to thank all staffs from Department of Pathology, Shanghai Cancer Center, Fudan University for their amazing work.

\section{Author Contributions}

Conceived and designed the experiments: GC. Performed the experiments: PW RW. Analyzed the data: PW RW. Contributed reagents/materials/ analysis tools: PW RW. Contributed to the writing of the manuscript: PW RW DS HZ LH WG YX SG GC.

11. Pintor MP, Northover JMA, Nicholls RJ (1989) Squamous cell carcinoma of the anus at one hospital from 1948 to 1984 British Journal of Surgery Volume 76, Issue 8. British Journal of Surgery 76: 806-810.

12. Stearns MW Jr, Quan SH (1970) Epidermoid carcinoma of the anorectum. Surgery, gynecology \& obstetrics 131: 953-957.

13. Bebenek M, Wojnar A (2009) Infralevator lymphatic drainage of low-rectal cancers: preliminary results. Annals of surgical oncology 16: 887-892.

14. Tocchi A, Lepre L, Costa G, Liotta G, Mazzoni G, et al. (1999) Rectal cancer and inguinal metastases: prognostic role and therapeutic indications. Diseases of the colon and rectum 42: 1464-1466.

15. Mistrangelo M, Pelosi E, Bello M, Castellano I, Cassoni P, et al. (2010) Comparison of positron emission tomography scanning and sentinel node biopsy in the detection of inguinal node metastases in patients with anal cancer. International journal of radiation oncology, biology, physics 77: 73-78.

16. Tonouchi H, Ohmori Y, Kobayashi M, Konishi N, Tanaka K, et al. (2004) Operative morbidity associated with groin dissections. Surgery today 34: 413418.

17. Paley PJ, Johnson PR, Adcock LL, Cosin JA, Chen MD, et al. (1997) The effect of sartorius transposition on wound morbidity following inguinal-femoral lymphadenectomy. Gynecologic oncology 64: 237-241.

18. Urist MM, Maddox WA, Kennedy JE, Balch CM (1983) Patient risk factors and surgical morbidity after regional lymphadenectomy in 204 melanoma patients. Cancer 51: 2152-2156.

19. Taylor N, Crane C, Skibber J, Feig B, Ellis L, et al. (2001) Elective groin irradiation is not indicated for patients with adenocarcinoma of the rectum extending to the anal canal. International journal of radiation oncology, biology, physics 51: 741-747.

20. Glimelius B, Pahlman L, Cervantes A, Group EGW (2010) Rectal cancer: ESMO Clinical Practice Guidelines for diagnosis, treatment and follow-up. Annals of oncology: official journal of the European Society for Medical Oncology/ESMO 21 Suppl 5: v82-86. 